\title{
Etiology of Bacteremic Syndromes and Bacterial Susceptibility of Blood Culture Isolates in a Romanian County Hospital
}

\author{
Man $\mathrm{A}^{1,2}$, Muntean $\mathrm{PE}^{3}$, Mare Anca ${ }^{1}$ \\ ${ }^{1}$ Department of Microbiology, University of Medicine and Pharmacy, Tîrgu Mureș, Romania \\ 2 Central Laboratory, County Emergency Clinical Hospital, Tîrgu Mureș, Romania \\ 3 Pneumology Clinic, County Emergency Clinical Hospital, Tîrgu Mureș, Romania
}

\begin{abstract}
Aim: To present the etiology of bacteremic syndromes and antibiotic susceptibility of blood culture isolates from a Romanian county hospital, as well as their distribution within different wards.

Methods: We retrospectively analyzed the blood culture data collected from patients hospitalized in the County Emergency Clinical Hospital of Tirgu Mures over a period of two years. We followed aspects regarding the identified bacterial species, their distribution by sex, age groups and wards, the spectrum of resistance to antibiotics and main resistance phenotypes.

Results: Most positive samples came from ICU, nephrology and urology. The most isolated bacteria were coagulase-negative staphylococci, Escherichia coli, and Staphylococcus aureus. All isolates showed a high resistance to most classes of antibiotics, staphylococci being susceptible to glycopeptides, oxazolidinones and glycylcyclines, and the enterobacteria to aminoglycosides and carbapenems. The resistance in non-fermentative bacilli exceeded $80 \%$ to most classes of antibiotics. The methicillin-resistance was 36\% for coagulase-negative staphylococci and $82 \%$ for Staphylococcus aureus; the percentage of extended-spectrum beta-lactamase producing strains was 30\%.

Conclusions: The etiology of bacteremic syndromes is specific to the ward profile, the Staphylococcus spp. being primarily isolated from wards where invasive procedures are frequently performed, while the enterobacteria from urology and nephrology wards. The level of antibiotic resistance is higher in surgery and ICU wards, with also higher percentage of resistance phenotypes than in medical wards.
\end{abstract}

Keywords: sepsis, blood cultures, blood culture contaminants, antibiotic susceptibility, resistance phenotypes

Received: 16 December 2013 / Accepted: 25 May 2014

\section{Introduction}

Although great progress has been made in recent years in medical research, the problem of serious infections with progression to sepsis remains incompletely elucidated. Bacteremic syndromes (presence of bacteria in the blood stream, associated with fever, chills, shortness of breath, though it may cause no symptoms) are currently a major cause of morbidity and mortality, therefore etiologic and epidemiologic studies must be considered. Furthermore, the misuse and the abuse of broad-spectrum antibiotics in hospitals is favoring the selection of resistant strains to antibiotics and chemotherapeutics [1,2]. Sepsis remains a major problem in intensive care units (ICU), with an estimated cost of $€ 20,000-€ 30,000 /$ case in Europe, thereby with a strong financial impact on each country's health budget [3].

Hospitalized people present multiple predisposing factors for sepsis, such as poor immunological status, trauma, invasive devices or certain pathological conditions, and types of treatments that increase vulnerability to infections. In addition, patients colonized at admission are exposed to a greater risk of infection when invasive procedures are applied [4-7].
The microbiological evidence of sepsis is sometimes deficient. Blood cultures are often negative, and results of microbiological investigations can be misleading in many cases $[8,9]$. The interpretation of microbiological results for patients in critical condition can be difficult, as a differentiation between contamination and infection cannot be possible sometimes, especially if more samples are not simultaneously and correctly collected [10].

\section{Purpose}

Evaluation of bacterial etiology of diseases associated with bacteremia in patients hospitalized in the County Emergency Clinical Hospital of Tîrgu Mureș, Mureș county, Romania, following the distribution of different bacterial species in medical and surgical wards and the evaluation of antibacterial resistance spectrum of the blood culture isolates.

\section{Material and methods}

In this retrospective study, we analyzed the blood culture data from patients hospitalized in the County Emergency Clinical Hospital of Tîrgu Mureș between October 18, 2010 and October 18, 2012. We followed aspects regarding the variability of bacterial species, their distribution by sex, age groups and wards, the spectrum of resistance to antibiotics, resistance phenotypes that are common in 
practice (methicillin-resistant staphylococci, extended spectrum beta-lactamase producing enterobacteria).

The blood cultures were collected under the suspicion of a bacteremic syndrome and processed by the bioMérieux BactAlert ${ }^{\circ}$ automated blood culture system; for identification of bacteria, both conventional methods and automatic bioMérieux Vitek2 ${ }^{\circ}$ system were used, according to the laboratory's standard working procedures. Data were collected from blood culture registers, subsequently pooled and processed using spreadsheet software. To avoid statistical errors, we considered only the first isolate of the same species isolated for each patient. If multiple blood cultures were processed from the same patient, and the results regarding the bacterial species and antibiotic susceptibility were similar, we counted the data only once. Otherwise, each different blood culture was counted separately.

Statistical analysis was performed using GraphPad InStat 3.0 program, with an alpha significance level of 0.05 .

The study was conducted with the approval of Ethics Committee for scientific research of the University of Medicine and Pharmacy of Tîrgu Mureș, all patient data being anonymized.

\section{Results}

The microbiology laboratory from where the records were analyzed, processed samples from intensive care units (ICU), surgical departments (general surgery, urology), medical (medical clinic, nephrology, pneumology, gastroenterology) and pediatric wards. During the study, 1548 blood cultures were collected, and 123 were positive cases (7.95\%). The age group from which came most of the bacterial isolates was $60-69$ years ( 41 cases, 33.3\%), followed closely by $70-79$ years (30 cases, $24.4 \%$ ), with a median of 64 years, where predominantly staphylococci (26 isolates, $21.1 \%$ ) and enterobacteria (30 isolates, 24.4\%) were found.

Looking at the gender distribution there was a male predominance of positive samples (74 cases, $60.1 \%)$ as

Table I. Distribution of microbial isolates by wards

\begin{tabular}{|c|c|c|c|c|c|c|c|c|}
\hline Wards & 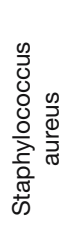 & 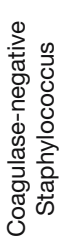 & 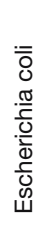 & 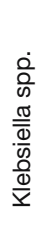 & 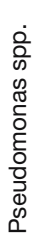 & 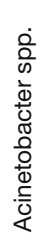 & 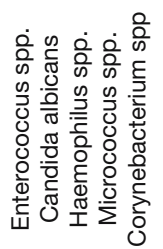 & $\frac{\frac{1}{\sigma}}{\circ}$ \\
\hline ICU & 6 & 19 & 4 & 2 & & 7 & 4 & 42 \\
\hline Nephrology & 4 & 7 & 10 & 1 & & & 6 & 28 \\
\hline Urology & & 2 & 11 & 4 & 2 & & 1 & 20 \\
\hline Medical clinic & 4 & 3 & 4 & 2 & & 1 & & 14 \\
\hline Surgery clinic & 2 & 2 & 1 & 1 & 2 & 1 & & 9 \\
\hline Pediatrics & 1 & 1 & & 1 & & & & 3 \\
\hline Pneumology & & 2 & 1 & & & & & 3 \\
\hline Gastroenterology & & & & & & 1 & 1 & 2 \\
\hline Neurology & & & & 1 & & & & 1 \\
\hline Burn ward & & & & & 1 & & & 1 \\
\hline TOTAL & 17 & 36 & 31 & 12 & 5 & 10 & 12 & 123 \\
\hline
\end{tabular}

compared to females (49 cases, 39.9\%). Coagulase-negative staphylococci (CoNS) were identified in the highest percentage $(29.3 \%)$, closely followed by Escherichia coli $(25.2 \%)$. A smaller percentage was represented by Staphylococcus aureus (13.8\%), the rest of the species being found in lower percentages, between $0.8 \%$ and $9.8 \%$.

Most positive samples were from the ICU ward, followed by nephrology and urology (Table I).

Among the species of staphylococci, most (68\%) were CoNS (Staphylococcus epidermidis and hominis, 16 cases in each one, the rest being represented by Staphylococcus haemolyticus, cohni and simulans). Staphylococcus aureus was identified in lower percentage (32\%).

Staphylococci showed a high resistance (over 60\%) to most classes of antibiotics, retained sensitivity to vancomycin, linezolid and tigecycline. There was a good sensitivity to trimethoprim-sulfamethoxazole for Staphylococcus aureus, of $86.7 \%$. The methicillin-resistance was $82 \%$ for Staphylococcus aureus and 36\% for CoNS (Figure 1).

Distribution by wards show a preponderance of isolates from ICU for both Staphylococcus aureus (6 cases, 35.3\% of all Staphylococcus aureus isolates) and the CoNS (19 cases; $52.7 \%$ of all CoNS). Although in some wards the number of staphylococcal isolates was small, the methicillin-resistance reached up to $100 \%$ for Staphylococcus aureus (nephrology, pediatrics, surgery). High proportion of methicillin-resistance $(68.4 \%)$ was seen in CoNS in ICU ward.

The statistical comparison regarding the resistance degree to antibiotics, show significant differences only for ciprofloxacin, in case of CoNS ( $p=0.025)$, with increased resistance in surgical wards and ICU (87\%) compared to the medical wards (46.2\%). For other classes of antibiotics, although the level of resistance is higher in surgical wards and ICU than in medical wards, the differences were not statistically significant. MRSA was found in higher percent in medical wards (88.9\%) (Figure 2).

The number of staphylococcal strains isolated from both aerobic blood culture bottle and also in the anaerobic was significantly higher in $S$. aureus compared with CoNS, with $\mathrm{p}<0.0001, \mathrm{OR}=2.676(1.399-5.119$ at CI 95\%).

Within the fermentative Gram-negative bacilli, two species were identified: Escherichia coli (72.09\%) and Kleb-

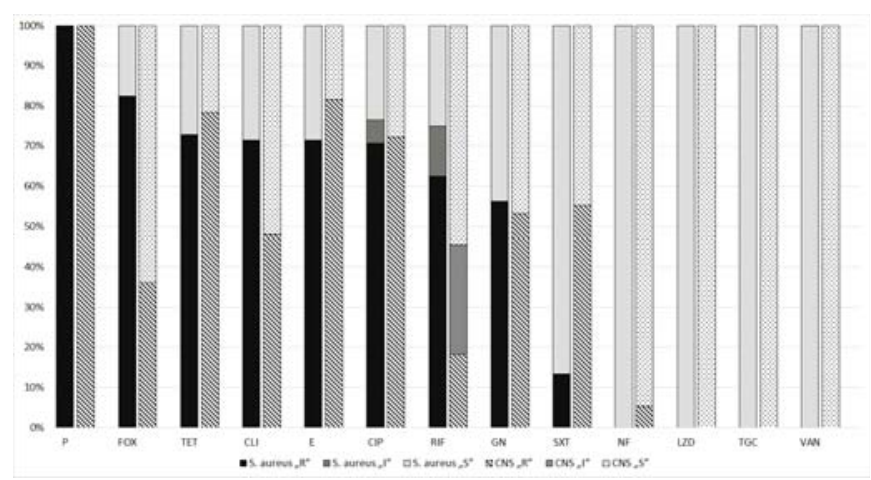

Fig. 1. Antibiotic susceptibility spectrum of staphylococci 


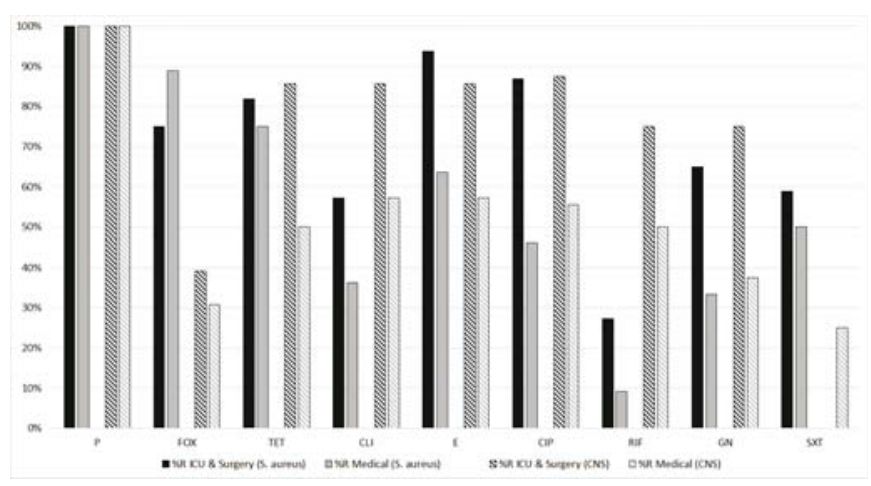

Fig. 2. Antibiotic resistance (\%R) among clinical ward types

siella pneumoniae (27.91\%). Out of the total, 13 strains of Enterobacteriaceae were identified as extended-spectrum beta-lactamase (ESBL) producing ones (30.23\%).

A larger number of Enterobacteriaceae (30 cases, 70\%) were isolated from the male gender than from females, and the elderly (60-80 years) were most involved. In Enterobacteriaceae, a medium level of resistance to most classes of antibiotics was observed. There was good sensitivity to aminoglycosides and carbapenems (Figure 3).

Most isolates belonging to Enterobacteriaceae were derived from urology wards (15 cases, 34.88\%) and nephrology (11 cases, $25.58 \%)$. In other surgical wards except urology, fewer enterobacteria were identified.

The degree of antibiotic resistance was generally higher in the wards that are using invasive procedures than in medical wards, for all classes of antibiotics except tetracycline and gentamicin. Significant differences occurred only in the case of fluoroquinolones, with a much higher resistance $(81 \%)$ in surgical wards and ICU compared to the medical ones (40\%), $\mathrm{p}=0.017$. The percentage of ESBL producing strains was $30.2 \%$, with no significant differences between the two types of wards $(\mathrm{p}=0.738)$.

In the category of non-fermentative Gram-negative bacilli (NFGNB), Acinetobacter baumannii was mainly identified (10 cases, 67\%), followed by Pseudomonas aeruginosa (4 cases, 27\%) and Pseudomonas putida (1 case, $6 \%$ ). The age group most affected by NFGNB was between $60-69$ years ( 7 cases, $46.7 \%$ ). The highest number of NFGNB was identified in ICU ward (7 cases, $46.7 \%$, all of which were represented by Acinetobacter spp.).

The NFGNB resistance to antibiotics was extremely high, exceeding $80 \%$ in most classes of antibiotics. Medium strength (about 50\%) occurred to amikacin, tobramycin and imipenem. Very good sensitivity (100\%) was identified only to colistin.

Except for Candida albincans ( 3 cases, $0.02 \%$ ), no other fungal species were identified.

\section{Discussions}

In this study there was a low rate of positivity (8\%) as compared to the blood cultures described in the literature. For example, in an observational cohort study published in 2006 , the positivity rate was $60 \%$, and in a study in inten-

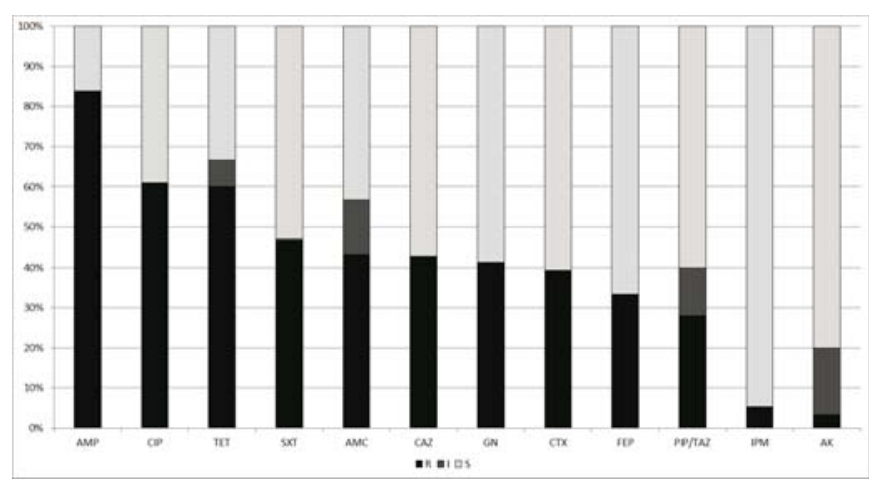

Fig. 3. Antibiotic susceptibility spectrum of enterobacteria

sive care units, the positivity rate was $50 \%$ only for CoNS $[11,12]$. Failure in respecting the clinical and laboratory criteria for collecting the blood cultures can lead to a low rate of positivity [13].

Most samples positive for staphylococci and NFGNB were collected from the ICU wards, and from urology in case of enterobacteria, respectively. In a similar study conducted in another clinical hospital in Tîrgu Mureș, the identified species and ward distribution were about the same to those presented in the current study, but for Staphylococcus aureus $64 \%$ were methicillin-resistant - a lower percentage than the one identified by us $(82 \%)$, and among Enterobacteriaceae predominated the Klebsiella spp. (47\%); the percent of ESBL producing enterobacteria was about the same (31\%) [14].

In this study, staphylococci (Staphylococcus aureus in particular) presented a high level of antibiotic resistance, including methicillin-resistance, aspects described in other studies and reports, but usually at lower levels (MRSA between $1-50 \%$ in Europe, $49.5 \%$ in Romania in 2011, 44\% in a cohort study from USA) $[15,16]$. It can be concluded that the degree of methicillin-resistance of staphylococci is high in our area.

The high percentage of Escherichia coli isolates in males can be explained by the large number of samples obtained from the urology ward, knowing that this bacterial species is commonly involved in urinary tract infections [17].

In this study, the NFGNB were identified in surgical and ICU wards, and their resistance to antibiotics was extremely high, even higher than $80 \%$ in most classes of antibiotics. Similar findings have been identified in other studies, such as a recent one from 2012 , where $22 \%$ of the isolates were from ICU wards; in contrast, Acinetobacter baumannii general resistance to carbapenems was $90 \%$, almost double to the one identified in this study [18]. According to other data, resistance to carbapenems can be quite low, only about $4.5 \%$ [19].

The differentiation between contamination and etiological involvement is still a problem for blood cultures [20]. A study of 843 positive blood cultures managed to differentiate the germs that are commonly involved in real bacteremic syndromes from those that are frequently contaminants. In the first category were included Staphylo- 
coccus aureus, Streptococcus pneumoniae, Escherichia coli and other enterobacteria, Pseudomonas aeruginosa and Candida albicans, but also other germs with definite, but rare involvement, like beta hemolytic streptococci (Streptococcus pyogenes and agalactiae), Neisseriaceae (Neisseria meningitidis and gonorrhoeae), Haemophilus influenzae or Listeria monocytogenes). The second category, which means contamination, included CNS, Corynebacterium spp., Bacillus spp., Propionibacterium acnes, Micrococcus spp., viridans group streptococci and enterococci [21].

In our case, the CoNS situation is particularly noticed, as being part of the normal skin flora, they may frequently be contaminants of the samples; other possible contaminants were also identified (Corynebacterium spp., Micrococcus spp., Enterococcus spp.). The same situation was also described in other clinics. For example, several studies have identified the CoNS as the most frequent isolates, but in much smaller percentage than us, and yet, questions about the collection and identification techniques have been raised $[22,23]$.

However, there is no denying the fact that CoNS can be involved in infections, especially in those related to intravascular devices, including sepsis. We could not clarify the relevancy of CoNS isolates, because in the absence of twin sets of blood culture collection, we were not able to follow the diagnostic criteria for CoNS involvement in bacteremia. In a recent study it was determined that the percentage of contaminated blood cultures is quite high (41\%), only $51 \%$ representing real infection, and of the identified CoNS, only $10 \%$ were found to be involved in the etiology of bacteremic syndrome [24]. Other studies established that the real etiologic agents in sepsis that are most frequently encountered are Staphylococcus aureus and E. coli [25]. We noticed that the identification of CoNS was predominantly from aerobic bottles, whilst $S$. aureus was isolated both from aerobic and anaerobic bottles. This can be explained by the significantly favorable growth of Staphylococcus aureus in anaerobic conditions compared to CoNS [26], but can also be due a contamination of aerobic blood cultures with CoNS.

The antibiotic resistance of bacteria is generally high in our study. The misuse of broad-spectrum antibiotics is probably the most important factor that induced the multi-resistant germs as generalized infections etiologic agents, therefore several measures must be implemented: antibiotics must be used only when strictly necessary, in appropriate dose and duration [27]. The general opinion is that the initial treatment of serious infections is vital and frequently requires the use of broad-spectrum antibiotics, but it is equally important to determine the actual etiology of infection and the antibiotic susceptibility of isolated strains, so the antimicrobial treatment can be downgraded, and thereby to reduce the selective pressure exercised by broad spectrum antibiotics on bacteria [28]. If the condition of the patient allows this, a proper downgrade to oral antibiotics can be considered; this allows the outpatient treatment and further reduces the costs by reducing the length of hospitalization [29]. At the same time, enforcing an appropriate hand hygiene of medical staff is a valuable method for preventing the spread of microorganisms, including hospital-related ones as MRSA [30]. The hand washing does not remove all local bacteria, as the resident flora is strongly attached to the skin; nevertheless, the pathogenic bacteria (S. aureus, Gram-negative bacteria, yeasts) that are associated with healthcare-related infections are commonly part of transient flora, and more susceptible to hand washing [31]. Thus, this simple gesture can significantly decrease the rate of nosocomial sepsis [32].

\section{Conclusions}

The most common bacterial species identified from blood cultures are coagulase-negative staphylococci, but with reserves in their interpretation as etiological agents in bacteremic syndromes, followed by Escherichia coli, Staphylococcus aureus, Klebsiella pneumoniae and Acinetobacter spp. The etiology of bacteremic syndromes is specific to the ward profile, the Staphylococcus spp. being primarily isolated from wards where invasive procedures are frequently performed, while the enterobacteria from urology and nephrology wards. The level of antibiotic resistance is a real concern, especially in surgery and ICU, especially as the specific resistance phenotypes are present and high. Some of the few classes of antibiotics that can be successfully used in the treatment of bacteremic syndromes are "reserve" considered ones, such as vancomycin, linezolid or imipenem.

\section{Acknowledgments}

The study was conducted within the project nr. 31/16432/11.12.2013 of the University of Medicine and Pharmacy Tîrgu Mureș Internal Competition for Research Grants.

\section{Abbreviations}

AK Amikacin

AMC Amoxicillin/Clavulanic acid

AMP Ampicillin

CAZ Ceftazidime

CIP Ciprofloxacin

CLI Clindamycin

CoNS Coagulase-negative staphylococci

CTX Cefotaxime

E Erythromycin

ESBL extended-spectrum beta-lactamase

FEP Cefepime

FOX Cefoxitin

GN Gentamicin

ICU Intensive care unit

IPM Imipenem

LZD Linezolid

MRSA Methicillin-resistant Staphylococcus aureus

NF Nitrofurantoin 
NFGNB Non-fermentative Gram-negative bacilli

$\mathrm{P} \quad$ Penicillin

PIP/TAZ Piperacillin/Tazobactam

RIF Rifampicin

SXT Trimethoprim/Sulfamethoxazole

TET Tetracycline

TGC Tigecycline

VAN Vancomycin

\section{References}

1. English BK, Gaur AH. The use and abuse of antibiotics and the development of antibiotic resistance. Adv Exp Med Biol. 2010;659:73-82.

2. Martin GS, Mannino DM, Eaton S, Moss M. The epidemiology of sepsis in the United States from 1979 through 2000. N Engl J Med. 2003;348:154654.

3. Burchardi $\mathrm{H}$, Schneider $\mathrm{H}$. Economic aspects of severe sepsis: a review of intensive care unit costs, cost of illness and cost effectiveness of therapy. PharmacoEconomics. 2004;22:793-813.

4. Wang HE, Shapiro NI, Griffin R, et al. Chronic Medical Conditions and Risk of Sepsis. PLoS ONE. 2012;7:e48307.

5. Su J, Tsun A, Zhang L, et al. Preoperative Risk Factors Influencing the Incidence of Postoperative Sepsis in Human Immunodeficiency Virus-Infected Patients: A Retrospective Cohort Study. World J Surg. 2013;37:774-9.

6. Wafaisade A, Lefering R, Bouillon B, et al. Epidemiology and risk factors of sepsis after multiple trauma: An analysis of 29,829 patients from the Trauma Registry of the German Society for Trauma Surgery*: Crit Care Med. 2011;39:621-8.

7. Schmid H, Romanos A, Schiffl H, Lederer SR. Persistent nasal methicillinresistant staphylococcus aureus carriage in hemodialysis outpatients: a predictor of worse outcome. BMC Nephrol. 2013;14:93.

8. Bates DW, Sands K, Miller E, et al. Predicting bacteremia in patients with sepsis syndrome. Academic Medical Center Consortium Sepsis Project Working Group. J Infect Dis. 1997;176:1538-51.

9. Ranawaka UK, Rajindrajith EGDS, Perera KVHKK, et al. Clinical profile and difficulties in diagnosis of central nervous system infections in adult patients in a tertiary care hospital. Ceylon Med J. 2013;58:26-8.

10. Zafer Mengeloglu F, Tas T, Demırcan F. Comparison of contamination in simultaneous blood cultures with clinical findings. Med Glas Zenica. 2013;10:151-3.

11. Vincent J-L, Sakr Y, Sprung CL, et al. Sepsis in European intensive care units: results of the SOAP study. Crit Care Med. 2006;34:344-53.

12. Savithri MB, Iyer $V$, Jones $M$, et al. Epidemiology and significance of coagulase-negative staphylococci isolated in blood cultures from critically ill adult patients. Crit Care Resusc. 2011;13:103-7.

13. Previsdomini M, Gini M, Cerutti B, Dolina M, Perren A. Predictors of positive blood cultures in critically ill patients: a retrospective evaluation. Croat Med J. 2012;53:30-9.

14. Man A, Szekely E, Toma F, Mare A, Sipos A. Etiologia bacteriană în sindromul bacteriemic. Farmacorezistența germenilor izolați din hemoculturi. Satu Mare - Stud Și Comunicări Ser Științele Nat. 2007;8:245-8.

15. Shurland S, Zhan M, Bradham DD, Roghmann M-C. Comparison of mortality risk associated with bacteremia due to methicillin-resistant and methicillin-susceptible Staphylococcus aureus. Infect Control Hosp Epidemiol. 2007;28:273-9.
16. Antimicrobial resistance interactive database (EARS-Net) [Accesed September 21, 2013]. Available at: http://www.ecdc.europa.eu/en/ healthtopics/antimicrobial_resistance/database/Pages/database.aspx.

17. Linhares I, Raposo T, Rodrigues A, Almeida A. Frequency and antimicrobial resistance patterns of bacteria implicated in community urinary tract infections: a ten-year surveillance study (2000-2009). BMC Infect Dis. 2013;13:19

18. Namita J, Pushpa S, Lalit S. Acinetobacter baumannii isolates in a tertiary care hospital: Antimicrobial resistance and clinical significance. J Micro Infect Dis. 2012;2:57-63.

19. Rahbar M, Mehrgan H, Aliakbari NH. Prevalence of antibiotic-resistant Acinetobacter baumannii in a 1000-bed tertiary care hospital in Tehran, Iran. Indian J Pathol Microbiol. 2010;53:290-3.

20. Rahkonen M, Luttinen S, Koskela M, Hautala T. True bacteremias caused by coagulase negative Staphylococcus are difficult to distinguish from blood culture contaminants. Eur J Clin Microbiol Infect Dis Off Publ Eur Soc Clin Microbiol. 2012;31:2639-44.

21. Weinstein MP, Towns ML, Quartey SM, et al. The clinical significance of positive blood cultures in the 1990s: a prospective comprehensive evaluation of the microbiology, epidemiology, and outcome of bacteremia and fungemia in adults. Clin Infect Dis Off Publ Infect Dis Soc Am. 1997;24:584-602.

22. Karunakaran R, Raja NS, Ng KP, Navaratnam P. Etiology of blood culture isolates among patients in a multidisciplinary teaching hospital in Kuala Lumpur. J Microbiol Immunol Infect Wei Mian Yu Gan Ran Za Zhi. 2007;40:432-7.

23. Zenebe T, Kannan S, Yilma D, Beyene G. Invasive Bacterial Pathogens and their Antibiotic Susceptibility Patterns in Jimma University Specialized Hospital, Jimma, Southwest Ethiopia. Ethiop J Health Sci. 2011;21:1-8.

24. Pien BC, Sundaram $P$, Raoof $N$, et al. The clinical and prognostic importance of positive blood cultures in adults. Am J Med. 2010;123:81928.

25. Weinstein MP, Murphy JR, Reller LB, Lichtenstein KA. The clinical significance of positive blood cultures: a comprehensive analysis of 500 episodes of bacteremia and fungemia in adults. II. Clinical observations, with special reference to factors influencing prognosis. Rev Infect Dis. 1983;5:54-70.

26. Riley JA, Heiter BJ, Bourbeau PP. Comparison of Recovery of Blood Culture Isolates from Two BacT/ALERT FAN Aerobic Blood Culture Bottles with Recovery from One FAN Aerobic Bottle and One FAN Anaerobic Bottle. J Clin Microbiol. 2003;41:213-7.

27. Schito GC. The importance of the development of antibiotic resistance in Staphylococcus aureus. Clin Microbiol Infect. 2006;12 Suppl 1:3-8.

28. Lode $H$. Management of serious nosocomial bacterial infections: do current therapeutic options meet the need? Clin Microbiol Infect. 2005; $11: 778-87$.

29. Desai M, Franklin BD, Holmes AH, et al. A new approach to treatment of resistant gram-positive infections: potential impact of targeted IV to oral switch on length of stay. BMC Infect Dis. 2006;6:94.

30. Kim YC, Kim MH, Song JE, et al. Trend of methicillin-resistant Staphylococcus aureus (MRSA) bacteremia in an institution with a high rate of MRSA after the reinforcement of antibiotic stewardship and hand hygiene. Am J Infect Control. 2013;41:e39-43.

31. Guideline for Hand Hygiene in Health-Care Settings. Recommendations of the Healthcare Infection Control Practices Advisory Committee and the HICPAC/SHEA/APIC/IDSA Hand Hygiene Task Force [Accesed May 18, 2014]. Available at: http://www.cdc.gov/mmwr/preview/mmwrhtml/ rr5116a1.htm.

32. Chhapola V, Brar R. Impact of an educational intervention on hand hygiene compliance and infection rate in a developing country neonatal intensive care unit. Int J Nurs Pract. 2014:Article first published online. 